\title{
The role of expectation in multisensory body representation - neural evidence
}

\author{
Francesca Ferri, ${ }^{1}$ Ettore Ambrosini, ${ }^{2}$ Paola Pinti, ${ }^{3}$ Arcangelo Merla ${ }^{3}$ and Marcello Costantini ${ }^{1,4,5}$ \\ ${ }^{1}$ Centre for Brain Science, Department of Psychology, University of Essex, Colchester, CO4 3SQ, UK \\ ${ }^{2}$ Department of Neuroscience, University of Padova, Padova, Italy \\ ${ }^{3}$ Infrared Imaging Lab, Institute for Advanced Biomedical Technologies - ITAB, Department of Neuroscience, Imaging and \\ Clinical Sciences, University G. d'Annunzio, Chieti-Pescara, Italy \\ ${ }^{4}$ Laboratory of Neuropsychology and Cognitive Neuroscience, Department of Neuroscience, Imaging and Clinical Sciences, \\ University G. d'Annunzio, Chieti, Italy \\ ${ }^{5}$ Institute for Advanced Biomedical Technologies - ITAB, University G. d'Annunzio, Chieti, Italy
}

Keywords: fNIRS, multisensory integration, rubber hand illusion, sensory prediction, sensory processes

\begin{abstract}
Sensory events contribute to body ownership, the feeling that the body belongs to me. However, the encoding of sensory events is not only reactive, but also proactive in that our brain generates prediction about forthcoming stimuli. In previous studies, we have shown that prediction of sensory events is a sufficient condition to induce the sense of body ownership. In this study, we investigated the underlying neural mechanisms. Participants were seated with their right arm resting upon a table just below another smaller table. Hence, the real hand was hidden from the participant's view and a life-sized rubber model of a right hand was placed on the small table in front of them. Participants observed a wooden plank while approaching - without touching - the rubber hand. We measured the phenomenology of the illusion by means of questionnaire. Neural activity was recorded by means of near-infrared spectroscopy (fNIRS). Results showed higher activation of multisensory parietal cortices in the rubber hand illusion induced by touch expectation. Furthermore, such activity was correlated with the subjective feeling of owning the rubber hand. Our results enrich current models of body ownership suggesting that our multisensory brain regions generate prediction on what could be my body and what could not. This finding might have interesting implications in all those cases in which body representation is altered, anorexia, bulimia nervosa and obesity, among others.
\end{abstract}

\section{Introduction}

A fundamental function of our brain is to generate predictions about upcoming events using prior information and contextual cues (Friston et al., 2006; Bubic et al., 2010; Friston, 2010). Such top-down probabilistic representations are plastic and constantly updated and affect the way we perceive and interact with the external world (Brown \& Brüne, 2012). Indeed, valid predictions allow optimization of bottom-up sensory processing (van Ede et al., 2010, 2011) and enhance behavioural responses to environment stimuli (Lunghi et al., 2014). For instance, top-down prediction of low-level features (e.g. colour, direction of motion) leads to facilitated processing of

Correspondence: F. Ferri, as above.

E-mail: fferri@essex.ac.uk

Received 1 March 2017, revised 18 June 2017, accepted 19 June 2017

Edited by Sophie Molholm

Reviewed by Silvio Ionta, Centre Hospitalier Universitaire Vaudois, Switzerland; Sohee Park, Vanderbilt University, USA; Simone Cutini, University of Padova, Italy

The associated peer review process communications can be found in the online version of this article. stimuli containing those features (Ball \& Sekuler, 1981; Corbetta et al., 1990; Saenz et al., 2002).

A still unanswered question is whether and, if so, to what extent the predictive features of the brain may also contribute to the plasticity of multisensory body representation (Blanke, 2012; Ehrsson, 2012). For instance, does the ability of the brain to generate probabilistic predictions of the upcoming events regarding the body (i.e. if an approaching object will hurt it) affect the experience of owning a body and its multisensory representation?

Answering this question is relevant for at least two reasons: first, it would improve our understanding of how bottom-up mechanisms (e.g. sensory perception) and top-down mechanisms (e.g. knowledge about one's body and its relation to events in the environment) interact to generate a coherent representation of the body. Second, it would reveal a new role of multisensory brain regions, that is to support changes in body representations due to probabilistic predictions.

Behavioural evidence from the literature on the rubber hand illusion (RHI) supports the idea that ability of the brain to generate probabilistic predictions of upcoming events has a role to play in the plasticity of our multisensory body representation (Ferri et al., 
2013, 2014). In the original version of the illusion (Botvinick \& Cohen, 1998; Tsakiris \& Haggard, 2005; Costantini, 2014; Tsakiris, 2016), after synchronous visuo-tactile stimulation of a rubber hand and the participant's hidden hand, participants reported feeling of ownership towards the former (Tsakiris \& Haggard, 2005; Costantini \& Haggard, 2007; Thakkar et al., 2011). Recently, we showed that the RHI can be induced even if tactile stimuli are merely expected, rather than experienced (Ferri et al., 2013, 2014; for different findings, see Guterstam et al., 2016; but see Ferri \& Costantini, 2016 for a commentary). In that study, participants observed the experimenter's hand while approaching - without touching - a rubber hand placed on a small table in front of them. Their real hand was hidden below the same table. We measured the phenomenology of the illusion by means of skin conductance response and questionnaire. Both measures indicated that participants experienced the illusion that the experimenter's hand was about to touch their hidden hand rather than the rubber hand, as if the latter replaced their own hand. Autonomic responses revealed that embodiment of the rubber hand occurred once the approaching hand entered participants' peripersonal space (PPS). This did not occur when the rubber hand was rotated by $180^{\circ}$ or replaced by a piece of wood. In a further study, we showed that mere exposure to the rubber hand was not enough to induce a full-blown embodiment of the RH (Ferri et al., 2013; Ferri \& Costantini, 2016).

Regarding the original version of the RHI, a theoretical explanation in terms of probabilistic predictions and prediction errors (Friston et al., 2006; Friston, 2010) has been recently proposed by Apps \& Tsakiris (2013). According to these authors, under synchronous multisensory stimulation between the participant's hand and the rubber hand, ownership would arise as the result of probabilistic predictions generated in multisensory areas to explain away the 'surprise', or prediction error, of seeing and feeling touch at different locations. Hence, within this theoretical framework, abstract supramodal representations of sensory input that are processed in multisensory cortices alter body perception and sense of ownership. Several multisensory brain regions including the temporo-parietal junction, the inferior parietal lobe, the anterior insula and the inferior frontal gyrus are activated (Azanon et al., 2010; Ionta et al., 2011a,b, 2014; Serino et al., 2013) when participants experience the classic rubber hand illusion (Ehrsson et al., 2004, 2005; Makin et al., 2007, 2008; Tsakiris et al., 2007; Olive et al., 2015).

Is it reasonable to expect that any of these multisensory regions play a role also when the RHI arises as an effect of anticipation of touch, rather than from the conflict of seeing and feeling touch at different locations?

In the RHI induced by tactile expectation (Ferri et al., 2013, 2014), prediction errors would be generated by anticipation, rather than actual perception, of sensory events on the participant's and the rubber hand. In this context, multisensory brain regions would generate probabilistic predictions that affect body ownership, based on prior information about the body as the target of touch events anytime external stimuli approach it (Apps \& Tsakiris, 2013). Interestingly, among the multisensory regions activated during the classic RHI, the parietal cortices have been shown to respond during anticipation of sensory consequences of actions (Halje et al., 2015; Rizzolatti \& Sinigaglia, 2016) and somatosensory (i.e. tickling) stimuli (Carlsson et al., 2000). Based on this evidence, suggestions have been made that sensory predictions and processing of actual sensory input are subserved by similar neural substrates. In sum, there are reasons to hypothesize that multisensory parietal regions play a role in the RHI induced by expected, besides felt, touch.
In this functional near-infrared spectroscopy (fNIRS) study, we sought to test this hypothesis and, thus, provide evidence, for the first time, that multisensory parietal regions support the role of sensory expectation in the plasticity of body representation. We employed fNIRS as it is suitable for more ecological applications, compared to fMRI (Cutini \& Brigadoi, 2014). Indeed, to perform valid body illusion tasks, such as the RHI, it is essential to have the participants sit in a comfortable and natural position, with a perfect real hand/rubber hand alignment, and able to have full control and a clear view of their limbs (Ionta et al., 2011b).

\section{Materials and methods}

\section{Participants}

Sixteen healthy naive participants (10 men, mean age 24.2 years, range 20-29), with normal or corrected-to-normal vision, took part in this study after their written informed consent was obtained. One participant was removed due to technical issues with the digitizing procedure (one male; see Arizono et al., 2016; Shimada et al., 2005 for a similar sample size). All participants had normal or correctedto-normal visual acuity and were right-handed. The study was approved by the Ethics Committee of the 'G. d'Annunzio' University of Chieti and was conducted in accordance with the ethical standards of the 2013 Declaration of Helsinki.

\section{Procedure}

The experimental apparatus and procedure were similar to those described previously (Ferri et al., 2013, 2014). In brief, participants sat in front of a table with their right arm placed on it in a relaxed position at a fixed point. A wooden frame $(80 \times 30 \times 20$, $\mathrm{L} \times \mathrm{W} \times \mathrm{H}$ ) was positioned over the table to both hide the participant's hand and support the experimental object. This object was either a realistic prosthetic rubber hand $(\mathrm{RH})$ or a wooden object (WO), a wooden block of approximately the same size of the $\mathrm{RH}$ and with handlike features. The two objects were presented in separate experimental blocks. The participants' hand and the viewed object were aligned on the vertical axis and were positioned at $20 \mathrm{~cm}$ from each other (Fig. 1).

The experimenter stood at the participant's right side, completely hidden from the participants' view behind a black curtain. He slowly introduced a wooden plank from behind the curtain into the participants' field of view and then moved it towards the viewed object with a constant velocity of $20 \mathrm{~mm} / \mathrm{s}(\mathrm{SD}<3 \mathrm{~mm} / \mathrm{s}$ as revealed by post hoc analysis on switch timing; Ferri et al., 2013), starting from a distance of $70 \mathrm{~cm}$ from the viewed object and stopping at $15 \mathrm{~cm}$ away from the viewed object; the wooden plank was then slowly removed from the participants' view (Fig. 1). Each approaching movement thus lasted approximately $30 \mathrm{~s}$. The wooden plank never touched the viewed object. The consistency of the experimenter's movements was assured by audio instructions delivered via earphones.

Four pairs of mechanical switches fixed onto two vertical rods enabled recording experimenter's movements, speed and position. The four pairs of switches were positioned on each rod at 60, 45, 30 and $15 \mathrm{~cm}$ from the viewed object. A further switch was located at the starting point $(70 \mathrm{~cm})$. The switch located at $30 \mathrm{~cm}$ allowed us to code the location of the approaching wooden block as being within either the participants' peripersonal space (PPS) or his/her extrapersonal space (EPS), that is when it was either $\leq 30 \mathrm{~cm}$ or $>30 \mathrm{~cm}$ from the viewed object. Although our main interest was in the distinction between PPS and EPS locations, we decided to use four 


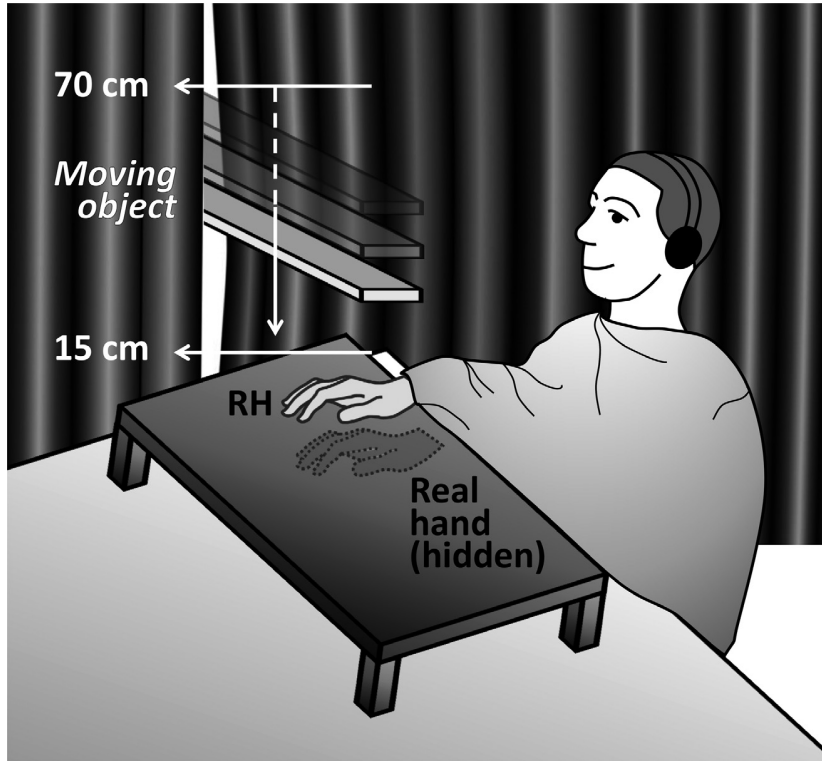

FIG. 1. Experimental set-up to evaluate rubber hand illusion. The experimenter moved a wooden plank towards the viewed object (which varied according to the experimental condition) with a velocity of $0.02 \mathrm{~m} \mathrm{~s}^{-1}$ starting from a distance of $70 \mathrm{~cm}$ from the viewed object and stopping at $15 \mathrm{~cm}$ away from it. The experimenter's hand never touched the viewed object. RH, rubber hand.

switches to better control for velocity of the approach, as changes in this parameter might increase arousal levels. Each switch sent a signal to a PowerLab system (ADInstruments), thus allowing post hoc validation of experimenter's movements and time-locking of experimenter's movements with the fNIRS signal.

The experiment consisted of two recording sessions - one for each hemisphere - separated by a short rest period during which the optical fibres were placed over the other hemisphere (see Functional near-infrared spectroscopy measurement). Each session consisted of two blocks (one for each experimental condition - RH and WO). The presentation order of both sessions and blocks within a session was counterbalanced between participants. Each block lasted approximately 5 min during which eight approaching movements were performed. The eight movements were interleaved by brief pauses $(\approx 15 \mathrm{~s})$. At the beginning of the experiment, participants were instructed to pay attention to the wooden plank moving towards the object placed in front of them (i.e. either the RH or the WO) and to avoid moving both their right arm and head. After receiving instructions, participants wore earphones through which white noise was delivered for the entire duration of each block. After each block, participants took off earphones and were required to complete a questionnaire (see Rubber Hand Illusion questionnaire).

\section{Rubber Hand Illusion questionnaire}

As we intended to replicate our previous findings (e.g. Ferri et al., 2013), we investigated whether expectation of touch experience arising at the sight of an object approaching a rubber hand was enough to induce in our participants a sense of body ownership over the same rubber hand, that is even when no physical tactile stimulation was applied on either the rubber hand or the participant's real hand. To this aim, we used the same questionnaire as in our previous works (Ferri et al., 2013, 2014) in a paper-and-pencil version.
In brief, 21 items taken from Longo et al. (Longo et al., 2008) assessed the four different components of the subjective experience of the rubber hand illusion: (i) the experience of embodiment of the $\mathrm{RH}$, assessed by eleven items related to the feelings that the $\mathrm{RH}$ belonged to the participant or had taken on features of the actual hand, that the RH and real hand were in the same location and that the participant had control over the RH; (ii) the experience of loss of one's hand, assessed by five items related to the feelings of being unable to move one's hand, and to the feeling of one's hand disappearing or being out of one's control; (iii) the feeling of movement, assessed by three items related to perceived motion of one's own hand, and to movement of the $\mathrm{RH}$; and (iv) the affect component, assessed by two items related to the appeal and enjoyment of the experience. Although the original questionnaire used by (Longo et al. (2008) included 27 items, we grouped only 21 items in clusters of experience.

Participants completed four versions of the questionnaire, one for each experimental block. They were asked to indicate the extent of their agreement or disagreement with the 21 statements using a 7point Likert scale. A response of -3 indicated strong disagreement with the statement, +3 strong agreement and 0 neither agreement nor disagreement.

\section{Functional near-infrared spectroscopy measurement}

Brain activity was recorded using a commercial frequency-domain near-infrared spectrometer (Imagent; ISS Inc., Champaign, IL, USA). The Imagent system was equipped with 32 laser diode sources (16 emitting at $690 \mathrm{~nm}$ and 16 at $830 \mathrm{~nm}$ ) modulated at $110 \mathrm{MHz}$ and four photomultiplier tube (PMT) detectors modulated at $110.005 \mathrm{MHz}$. The sources were time-multiplexed during recording (switch mode 32, one light active at once) resulting in a sampling frequency of $8.68 \mathrm{~Hz}$. Low-NIR-attenuation silica optical fibres were used to inject the light emitted by the diodes into the scalp. Back-scattered light was collected through 3-mm-diameter fibre-optic bundles connected to the PMTs. The diode light power at the fibre end was $<4 \mathrm{~mW} / \mathrm{cm}^{2}$, within the limits of the American National Standards Institute and thus permitting safe measurements.

The front ends of the optical fibres (optodes) were arranged on a custom-built rubber holder (recording pad) that was attached to the participants' head using Velcro strips. The optodes' spatial configuration is shown in Fig. 2. The source-detector pairs were arranged in a nearest-neighbour geometry creating 24 source-detector combinations (measurements channels). The source-detector distance was set at $3 \mathrm{~cm}$ as in most of the fNIRS studies on adults (Okada et al., 1997; Boas et al., 2004; Shimada et al., 2005; Arizono et al., 2016). The recording pad was placed unilaterally over the parietal and dorsal posterior temporal regions of one hemisphere in separate recording sessions. To place the recording pad in a reliable way across all the participants, we adopted a neuronavigation procedure. Before the experiment, a T1-weighted MR scan was obtained from each participant using a Siemens 3 T scanner $(1 \times 1 \times 1 \mathrm{~mm}$, sagittal acquisition). We then identified on the individual's scalp two points of reference (for the temporo-parietal junction and the lateral part of the parieto-occipital sulcus) by coregistering reference scalp locations to individual MR images using a neuronavigation system (Fastrak Polhemus) running a SofTaxic software. The two points of reference were localized on each hemisphere according to individually determined well-defined anatomical landmarks. The temporoparietal junction was localized at the intersection of the posterior end of the Sylvian fissure, the inferior parietal lobule and the lateral occipital cortex. The parieto-occipital sulcus was localized on the 


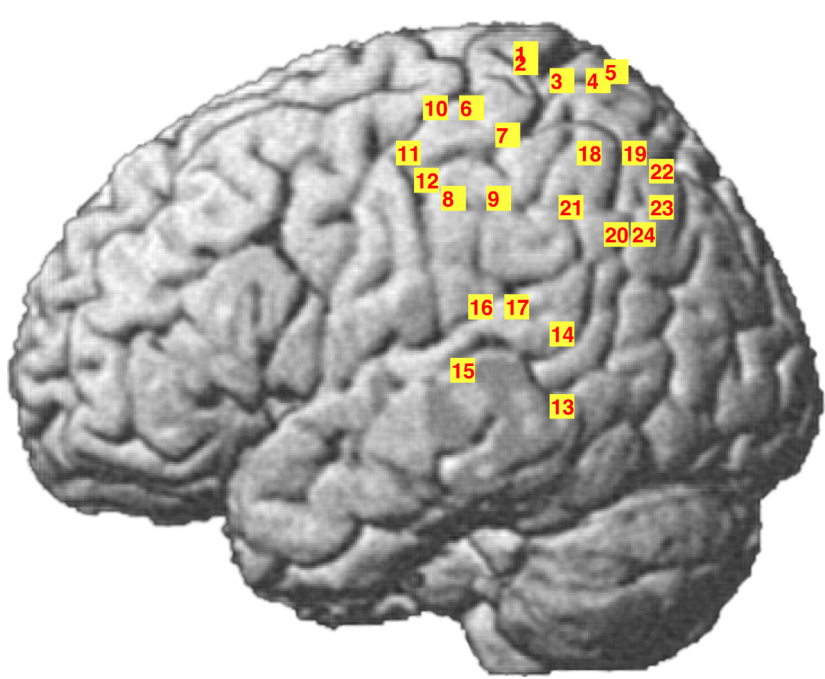

FIG. 2. Estimated cortical locations of the 24 NIRS channels (see Functional near-infrared spectroscopy measurement for details). [Colour figure can be viewed at wileyonlinelibrary.com].

lateral surface of each hemisphere just posteriorly to the posterior end of the intraparietal sulcus. The recording pad was thus placed according to these two anatomical landmarks on an individual basis to cover the posterior parietal cortex as well as the posterior temporal regions.

For each participant, the optodes' positions and the four cranial reference points (nasion, inion and preauricular points) were digitized using a 3D digitizer (Fastrak Polhemus). The digitized locations in real coordinates were then converted into the MNI space coordinates and localized onto the anatomical MR image using the four cranial reference points. The channels' spatial registration into the MNI space was performed using the NFRI toolbox (Okamoto \& Dan, 2005; Singh et al., 2005) in the NIRS-SPM software package (Ye et al., 2009).

\section{Functional near-infrared spectroscopy data analysis}

Data analysis of fNIRS signal was carried out separately for each hemisphere. Alternating current (AC), direct current (DC) and phase (Ф) components were computed for each channel and for each wavelength. The optical signal preprocessing was performed using the Homer2 NIRS Processing package (Huppert et al., 2009). The DC signals (light intensity) were converted into changes in optical density (OD) for each source-detector pair. After a visual inspection, motion artefacts were detected on the basis of the number of standard deviations (SDs) between the outlier points and the signal mean (Huppert et al.). Data points exceeding the threshold (i.e. 5 $\mathrm{SDs}$ ) in a time period of $1 \mathrm{~s}$ and points at $\pm 1 \mathrm{~s}$ from these outliers were marked as motion artefacts (Brigadoi et al., 2014). A channelbased cubic spline interpolation ( $p$ parameter $=0.99$ ) was employed to correct for motion artefacts (Scholkmann et al., 2010; Cooper et al., 2012). Signals were then band-pass-filtered (third-order Butterworth filter) with cut-off frequencies of $0.01-0.5 \mathrm{~Hz}$ to reduce physiological noises (e.g. heart beat) and slow drifts.

OD signals were converted into changes in concentration of oxyhaemoglobin $(\mathrm{HbO})$ and deoxyhaemoglobin $(\mathrm{HbR})$ using the modified Beer-Lambert law (Cope \& Delpy, 1988) with an age-corrected differential-pathlength factor (DPF) (Duncan et al., 1996). DPFs at 830 and $690 \mathrm{~nm}$ were set at 5.5 and 6 using the equations provided by Duncan et al. (1996). A correlation-based signal improvement
(CBSI) method (Cui et al., 2010; Brigadoi et al., 2014) was used to maximize the negative correlation between $\mathrm{HbO}$ and $\mathrm{HbR}$. To enhance any residual spikes, the carrier components of the concentration signals were subtracted from the time series and cubic spline interpolation was run again in case of large motion errors.

Single-subject and group analyses were then performed using the NIRS-SPM analysis package (Ye et al., 2009). To obtain corrected statistics, the precolouring method (Ye et al., 2009) was employed to swamp any intrinsic temporal correlation existing in fNIRS signals. This is achieved by smoothing the data with a low-pass filter shaped as the canonical haemodynamic response function (HRF) (Worsley \& Friston, 1995).

Possible significant neural activations in response to the approaching movements were assessed by means of a general linear model (GLM approach; Friston et al., 1994). In particular, the design matrix modelled the eight experimental events resulting from the combinations of the two experimental conditions (i.e. the viewed object - RH vs. WO) and the four distances of the approaching object from the viewed object (corresponding to the four switches located at $60,45,30$ and $15 \mathrm{~cm}$ from the viewed object; see Procedure). The transitions of the approaching object at the four distances were modelled as events using a stick function convolved with the HRF (Ye et al., 2009). The eight regressors were then compared to the $\mathrm{HbO}$ and $\mathrm{HbR}$ time series to yield appropriate parameter estimates of the experimental effects ( $\beta$-values).

Next, starting from the resulting $\beta$-values, we computed T-statistics corresponding to the contrasts of interest testing for the experimental effects relevant to our hypothesis. In doing this, the distance of the approaching object was defined dichotomically in relation to the switch located at $30 \mathrm{~cm}$ - the movement phase during which the approaching object was farther than $30 \mathrm{~cm}$ from the participants' hand (corresponding to the events for the 60 and $45 \mathrm{~cm}$ distances) was coded as EPS, while the movement phase during which the approaching object was closer than $30 \mathrm{~cm}$ from the participants' hand (corresponding to the events for the 30 and $15 \mathrm{~cm}$ distances) was coded as PPS. We did so to increase statistical power as in our previous work we showed that the autonomic response to a stimulus approaching the RH occurred only when the approaching stimulus entered the participant's PPS and it was not significantly different between 30 and $15 \mathrm{~cm}$, and between 60 and 45 (Ferri et al., 2013).

Therefore, the resulting four experimental conditions were as follows: (i) Rubber Hand - Extrapersonal Space (RH_EPS), in which the wooden plank approached the rubber hand but it was still in the participant's extrapersonal space (i.e. a distance larger than $30 \mathrm{~cm}$ from the RH); (ii) Rubber Hand - Peripersonal Space (RH_PPS), in which the wooden plank approaching the rubber hand has crossed the boundary of the participant's peripersonal space (i.e. a distance smaller than $30 \mathrm{~cm}$ from the RH); (iii) Wood Object - Extrapersonal Space (WO_EPS), in which the wooden plank approached the wooden object but it was in the participant's extrapersonal space; and (iv) Wood Object - Peripersonal Space (WO_PPS), in which the wooden plank approaching the wood object has crossed the participant's PPS boundary.

The resulting T-scores were then interpolated using the Gaussian random field interpolation (Ye et al., 2009), and activation maps were rendered onto a standard MNI brain template. In the group analysis, the subjects' interpolated maps were aligned in the MNI space and second-level statistics were computed for the intersection region of each subject's cortical area covered by the optodes. To control for false positives, all the resulting $P$-values were corrected by false discovery rate $($ FDR $<0.05$; Singh \& Dan, 2006). 


\section{Correlational analyses between $R H I$ questionnaire responses and $\mathrm{fNIRS}$ data}

We here aimed to investigate whether neural activity related to the RHI was linked to the participants' introspective ratings of their RHI experience. To assess the vividness of participants' RHI, differential RHI scores were measured by subtracting mean ratings provided after RH blocks from those provided after WO blocks for each of the components detailing the RHI experience. We then compared correlation coefficients (see Correlation between introspective evidence and fNIRS data) between these RHI scores and the $\beta$ values of the contrasts of interest for the NIRS channels surrounding regions of significant group activation based on the results of the GLM analyses.

\section{Results}

\section{Introspective evidence}

As described in Rubber Hand Illusion questionnaire, at the end of each of the four recording blocks the participants provided responses for a total of 21 items referring to four principal components of the subjective experience of the RHI: (i) Embodiment; (ii) Loss of one's own hand; (iii) Movement; and (iv) Affect.

First, the mean ratings for each component were submitted to a $2 \times 2$ repeated-measures ANOVA with the recording session (left vs. right parietal lobe) and the viewed object (RH vs. WO) as main factors. The distance between the approaching stimulus and the viewed cannot be used as a factor as participants filled in only one questionnaire after every approaching movement block. The ANovas led to similar results for all of the four principal components of the RHI experience. Indeed, the main effect of the viewed object was significant, with higher mean ratings for the $\mathrm{RH}$ condition compared to the WO condition (Embodiment: $F_{1,14}=52.66, \quad P<0.001$, $\eta_{\mathrm{p}}^{2}=0.79 ; \quad \mathrm{RH}=0.92, \quad \mathrm{WO}=-0.81 . \quad$ Loss: $\quad F_{1,14}=5.91$, $P=0.029, \quad \eta_{\mathrm{p}}^{2}=0.30 ; \quad \mathrm{RH}=-0.69, \quad \mathrm{WO}=-1.4 . \quad$ Movement: $F_{1,14}=9.09, \quad P=0.009, \quad \eta_{\mathrm{p}}^{2}=0.39 ; \quad \mathrm{RH}=-1.66, \quad \mathrm{WO}=-2.53$. Affect: $\quad F_{1,14}=11.79, \quad P=0.004, \quad \eta_{\mathrm{p}}^{2}=0.46 ; \quad \mathrm{RH}=1.07$, $\mathrm{WO}=-0.65$; see Fig. 3 ). These results showed that our paradigm was effective in inducing a more vivid experience of embodiment of the RH compared to WO in our participants. The main effect of the recording session and the two-way interaction were not significant (respectively, Embodiment: $F_{1,14}=0.12$ and $0.50, P=0.735$ and 0.493; Loss: $F_{1,14}=0.16$ and $1.18, P=0.696$ and 0.295 ; Movement: $F_{1,14}=2.40$ and $0.25, P=0.144$ and 0.624; Affect: $F_{1,14}=0.003$ and $0.16, P=0.959$ and 0.692$)$. The pattern of results was confirmed by a multivariate analysis of variance (viewed object: Wilks' $\Lambda=0.197 ; \quad F_{4,11}=11.19, \quad P<0.001$; recording session: Wilks' $\Lambda=0.766 ; \quad F_{4,11}=0.84, P=0.527$; two-way interaction: Wilks' $\Lambda=0.810 ; F_{4,11}=0.65, P=0.640$ ), as well as by nonparametric analyses (Wilcoxon signed-rank test; data not shown).

Next, we examined which component of the RHI experience was reliably elicited by our experimental manipulation. We thus performed a series of one-tailed one-sample $t$-tests against 0 on the main ratings of the four components assessed by the RHI questionnaire after each of the four experimental blocks. The analysis revealed that only Embodiment items consistently received mean ratings significantly higher than 0 in the RH condition [left hemisphere: $\quad M=0.91, \quad t(14)=2.07, \quad P=0.029, \quad d=0.54 ; \quad$ right hemisphere: $M=0.93, t(14)=2.23, P=0.021, d=0.58]$. Moreover, the Affect items received mean rating significantly higher than 0 in the right hemisphere session $[M=1.13, t(14)=2.26, P=0.02$,

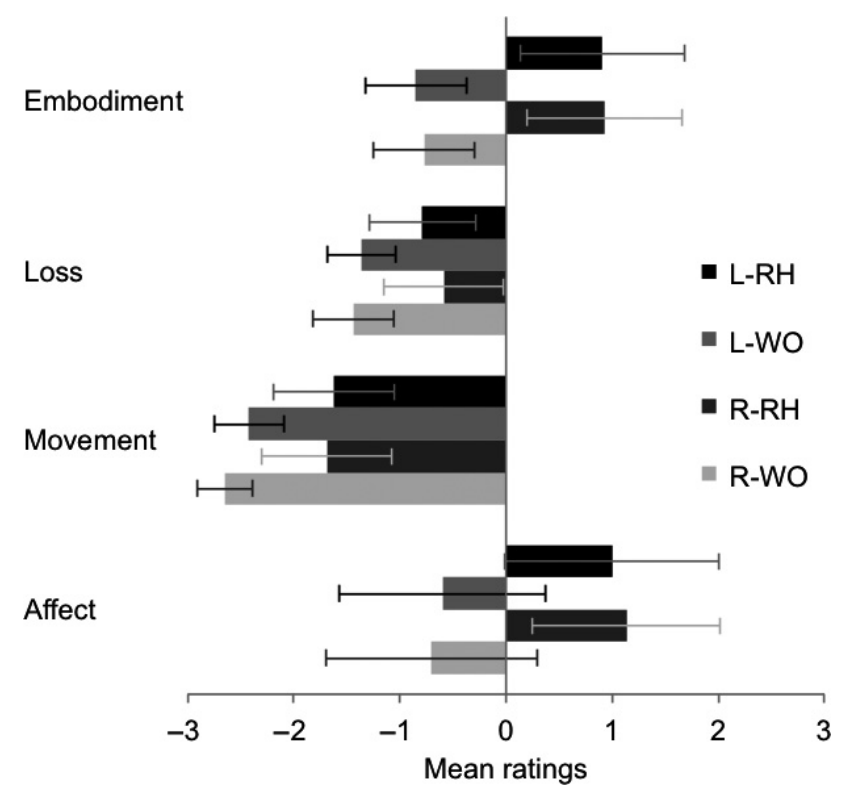

FIG. 3. Participants' mean ratings at the RHI questionnaire as a function of the RHI component and the experimental condition ( $\mathrm{L}$, left hemisphere; R, right hemisphere; RH, rubber hand; WO, wood object). Error bars represent $95 \%$ confidence intervals.

$d=0.58]$ but not in the left hemisphere session $[M=1.00, t$ $(14)=2.22, P=0.051, d=0.45]$. The mean ratings of all of the other variables were not significantly higher than 0 .

\section{Functional near-infrared spectroscopy results}

We aimed to reveal neural correlates of the sense of body ownership over a RH induced proactively by the expectation of touch from an approaching object. To this aim, we tested the linear contrast testing for the interaction effect that was relevant to our hypothesis [(RH_PPS-RH_EPS)-(WO_PPS-WO_EPS)].

The analysis on $\mathrm{HbO}$ revealed a distinct activation cluster in the inferior parietal lobule (IPL) in the left hemisphere $(P<0.05$, FDRcorrected; see Fig. 4a). This group activation cluster included the cortex surrounding the posterior end of the Sylvian fissure as well as the inferior parietal lobule and the temporo-parietal junction (see Fig. 4a). This result showed that an approaching object elicited significantly larger positive changes in $\mathrm{HbO}$ concentration when it fell within, rather than outside, the participant's PPS, and this differential activation was significantly more pronounced when the object approached the RH located just above the participant's hand, compared to when the same object approached the WO. Further supporting this finding, we ran the contrast (RH_PPS vs. RH_EPS) testing for the simple effect of the moving object location when approaching the RH. The result of this analysis revealed that significantly larger changes in $\mathrm{HbO}$ concentration $(P<0.05$, FDR-corrected) were elicited in the same cortical region when the object approaching the RH fell within, rather than outside, the participant's PPS. In contrast, the (WO_PPS-WO_EPS) contrast did not reveal significant activations.

The result of the $\mathrm{HbO}$ analysis was confirmed by the analysis on HbR change, which revealed a smaller cluster of significant group activation that overlapped the anterior part of the cluster emerged in the $\mathrm{HbO}$ analysis (see Fig. 4b). This result showed that the significant changes in $\mathrm{HbO}$ concentration revealed by the analysis reported above were closely mirrored by analogous significant changes in HbR concentration, both for the [(RH_PPS-RH_EPS)-(WO_PPS- 
(a)

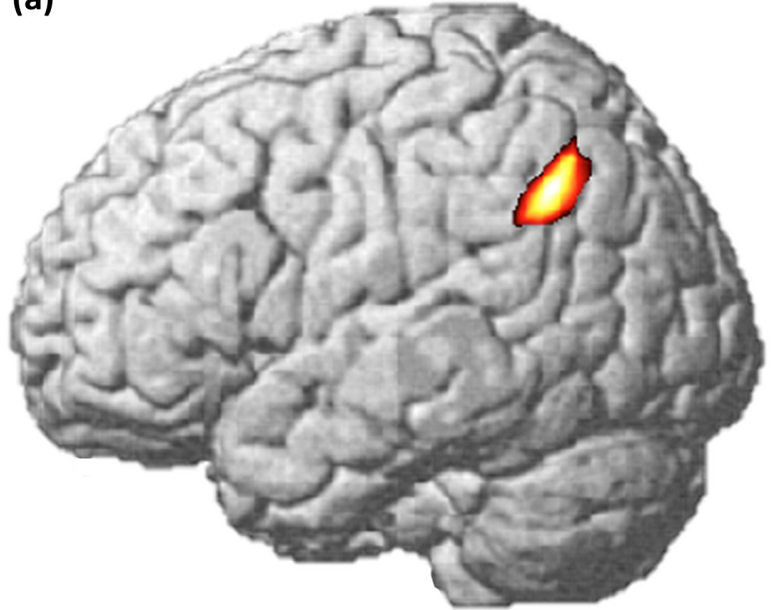

(b)

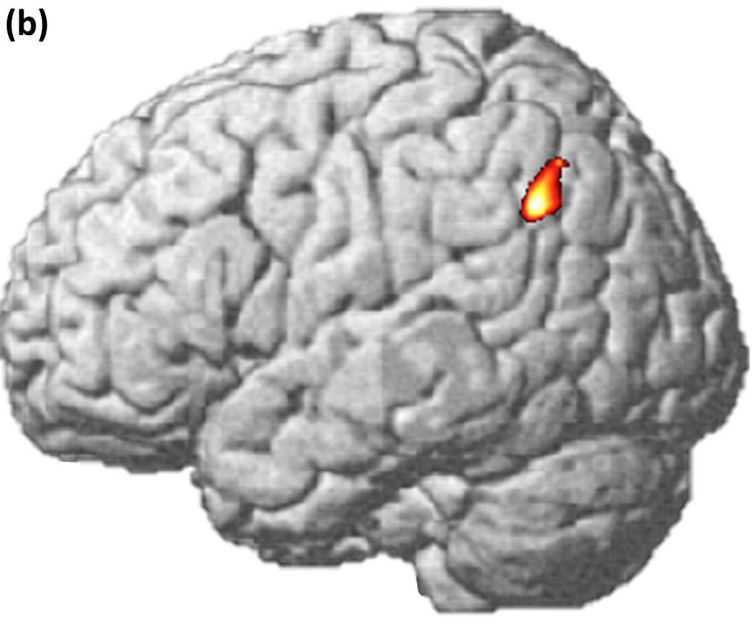

FIG. 4. Group activation T maps $(P<0.05$, FDR-corrected) for the (RH_PPS-RH_EPS)-(WO_PPS-WO_EPS) contrast on HbO (panel a) and HbR (panel b) fNIRS data. [Colour figure can be viewed at wileyonlinelibrary.com].

WO_EPS)] contrast testing for the interaction effect relevant to our hypothesis and for the (RH_PPS-RH_EPS) contrast testing for the simple effect of the moving object location in the RH condition (both $p s<0.05$, FDR-corrected). The smaller dimension of the cluster may be related to the fact that the magnitude of the HbR response is 2-3 times smaller than that of $\mathrm{HbO}$ response, resulting in a lower sensitivity but higher spatial selectivity for HbR signal changes (Plichta et al., 2007; Gagnon et al., 2012; Mihara et al., 2012).

The same analysis carried out for the right hemisphere did not reveal any significant group changes in either $\mathrm{HbO}$ or $\mathrm{HbR}$ (all $p s>0.05$, FDR-corrected).

To investigate the selectivity of touch anticipation on the rubber hand illusion in the peripersonal space, we tested the contrast [RH_EPS-WO_EPS]. No statistically significant differences were found for this contrast, in both hemispheres and for both chromophores (i.e. $\mathrm{HbO}$ and $\mathrm{HbR}$ ).

\section{Correlation between introspective evidence and fNIRS data}

We explored whether neural activity related to the proactive RHI was linked to the participants' introspective ratings of their RHI experience by means of a GLM analysis (separate-slopes model). To limit the number of statistic tests, we chose to restrict the analysis: (i) to the seven channels located more posteriorly (i.e. channels 1824; see Fig. 2), which were those that covered the cluster of significant group activation based on the results of the GLM analyses on $\mathrm{HbO}$ for the left hemisphere (see Functional near-infrared spectroscopy results); and (ii) to the corresponding mean ratings for the Embodiment component of the RHI questionnaire, which was the only relevant component reflecting the embodiment of the RHI that received reliable mean agreement ratings consistently (i.e. mean ratings significantly higher than 0 ; see Introspective evidence).

We thus carried out a separate-slopes analysis testing for significant across-channels differences in correlation (slopes) between the $\beta$-values for the [(RH_PPS-RH_EPS)-(WO_PPS-WO_EPS)] contrasts of interest and mean Embodiment RHI experience (see Correlational analyses between RHI questionnaire responses and fNIRS data). The analysis revealed the significance of the interaction term accounting for across-channels difference in the association link between $\beta$-values and mean Embodiment RHI $\left(F_{6,98}=2.85\right.$, $\left.P=0.013, \eta_{\mathrm{p}}^{2}=0.15\right)$. Post hoc correlational analyses corrected for multiple comparisons (Bonferroni-corrected $\alpha$ level: $0.05 / 7=$
0.007) revealed that the association link between $\beta$-values and mean Embodiment RHI (Pearson's correlation coefficient) was significant for the channel 21 only $[r=0.70, t(13)=3.58, P=0.003]$ (Fig. 5), that is the channel that more closely matched the location of peak significant activation in the group map (see Fig. 4).

Moreover, as correlation analyses are particularly sensitive to deviant observations (Rousselet \& Pernet, 2012), we confirmed this result by performing a robust correlation analysis. Specifically, we computed skipped parametric (Pearson) and nonparametric (Spearman) correlations (Wilcox, 2004) using the Robust Correlation toolbox (Pernet et al., 2012) and conducted null hypothesis statistical significance testing using the nonparametric percentile bootstrap test (2000 resamples; one-sided 95\% confidence interval, corresponding to an $\alpha$ level of 0.05 ), which is more robust against heteroscedasticity compared with the traditional $t$-test (Pernet et al., 2012; see also Ambrosini \& Vallesi, 2016). The results of the robust correlation analyses replicated the significant correlation between mean Embodiment RHI and $\beta$-values for the channel 21 only, thus showing that this result was not flawed by the presence of outliers or by the characteristics of our data.

\section{Discussion}

In a prior study (Ferri et al., 2013), we showed that people experience the illusion that a rubber hand, located just above their hidden

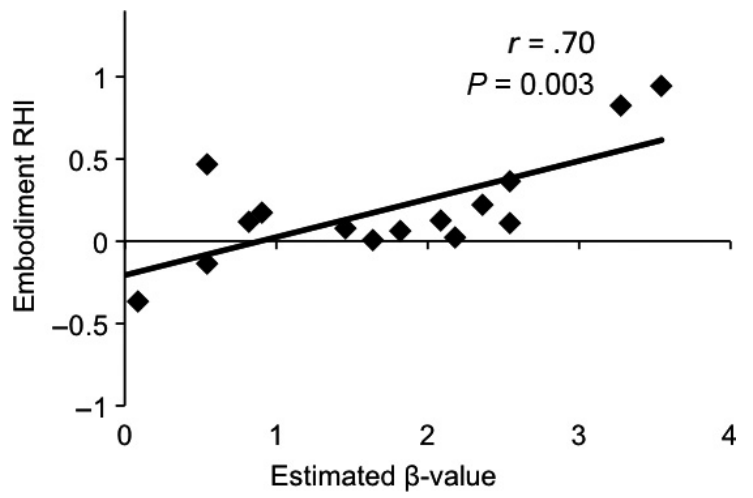

FIG. 5. Relation between the vividness of the RHI experience, as revealed by Embodiment statements ( $y$-axis), and the increase in BOLD signal ( $x$-axis, estimated $\beta$-value) for all participants. 
hand, is part of their body anytime an approaching object generates an expectation of touch. In that study, phenomenology of the illusion was measured by means of questionnaire and skin conductance response. We interpreted the RHI induced by touch expectation as an indication that our brain does not build a sense of self in a merely reactive way, via perceptual correlations; rather, it generates predictions on what may or may not belong to itself. The present study follows up and extends our prior work as it shows that the RHI induced by touch expectation relies upon activity in multisensory parietal cortices. Thus, for the first time, we reveal that parietal cortices critically support the role of sensory expectation in the plasticity of body representation.

We employed the fNIRS because it allowed us testing bodily self-representation in a natural position, as compared to the horizontal and unnatural position assumed within a magnetic resonance scanner. Hence, this also prevented any methodological confound that might interfere with the role of the vestibular system in bodily self-consciousness (Haggard et al., 2003; Blanke et al., 2004; Blanke \& Mohr, 2005; Lopez et al., 2008, 2012; Blanke, 2012). Indeed, vestibular signals are believed to contribute to bodily perceptions ranging from low-level (i.e. touch) to higher-level bodily perceptions (i.e. the sense of owning a body) (Lopez, 2015).

The present fNIRS study leads to two main new findings: (i) the contralateral inferior parietal cortex is selectively activated when an object approaching the rubber hand, rather than a piece of wood, falls within the participant's peripersonal space; and (ii) the activity in the left inferior parietal cortex predicts the individual proneness to experience the RHI evoked by tactile expectation, as specifically revealed by the correlation with subjective reports of the Embodiment of the rubber hand.

Interestingly, from a dynamic perspective, neural responses in the parietal cortices showed similar temporal patterns as previously exhibited by autonomic responses during the same task (Ferri et al., 2013) - both revealed that embodiment of the rubber hand occurs as soon as the approaching object has entered the participant's peripersonal space (PPS). Such similarity between dynamics of predictive neural and autonomic responses suggests a possible role of brain-body interaction in the prediction of external events. Future studies specifically testing this hypothesis are warranted.

All in all, our results are in agreement with the idea that during the RHI elicited by touch expectation, ownership would arise as the result of probabilistic predictions generated in multisensory parietal cortices - on the basis of prior information about the body as the target of approaching external stimuli - to explain away the prediction error of anticipating touch, on the seen rubber hand and the felt own hand.

But, what is the neuronal mechanism enabling the effect of an expected tactile stimulus on the perception of a delivered visual stimulus?

Single unit recordings in awake monkeys have shown that there are neurons in the bilateral parietal cortex with somatic anticipation responses (MacKay \& Crammond, 1987). This anticipatory activity was expressed as a change in discharge rate anytime a stimulus approaching a specific body part was presented. The same neurons also responded to somatosensory stimulation with a somatic receptive field corresponding to the region being approached. According to the authors' suggestions, a possible source of anticipatory information could be represented by the convergent projections that target the posterior parietal regions originating from sensory areas, prefrontal cortex, limbic areas
(Mesulam et al., 1977; Stanton et al., 1977), the pulvinar nuclei (Burbaud et al., 1985; Petersen et al., 1985) and the polysensory zone of the superior temporal sulcus (Seltzer \& Pandya, 1980) with neurons responding to somatic approach (or withdrawal) (Perrett et al., 1985). Drawing from their results, the authors argued that the approach response of parietal neurons signals the anticipation of a specific somatosensory stimulus. Specifically, the visual stimulus would serve to cue an internal model that predicts following events based on previously experienced associations (MacKay \& Crammond, 1987).

Anticipatory responses to tactile stimuli in the parietal cortices have been shown also in humans. Carlsson et al. (2000) found that brain activation in response to the expectation of a tactile stimulus, as a tickle provided with a light touch of painter's brush on the foot sole, is similar to the one engaged during actual somatosensory stimulation. Anticipation responses, however, are not specifically confined to the somatosensory domain. More recently, Kok et al. (2014), using fMRI, demonstrated that expectation of a visual stimulus induces an activation of the visual cortex which resembles the ones induced by the real stimulus. The mechanisms leading to the generation of anticipated responses seem to be related to low-frequency oscillatory activity of the brain and temporal regularity of the stimuli. Cravo et al. (2013) showed that the perception of the orientation of visual stimuli was modulated by the fact that the stimuli were embedded within temporally regular and predictable streams of noise patches, compared to irregular and unpredictable ones. At the neural level, the temporal regularities led to phase entrainment of slow oscillatory activity in anticipation of the expected event so that the stimulus occurred at the phase with optimal performance. Based on this evidence, we can speculate that in our study, the temporal regularity of the approaching stimuli might led to a similar phase entrainment of slow oscillatory activity leading to neural responses to the expected touch. In other words, during the new version of the rubber hand illusion, top-down expectations would interact with bottom-up somatosensory responses (occurring in the parietal cortex even in the absence of actual stimulation) via phase entrainment. Future research is warranted to test this hypothesis.

However, even if the specific mechanism involved is still to be clarified, the fact that the embodiment of the rubber hand elicited by touch expectation is predicted by anticipatory response in multisensory regions (i.e. the parietal cortex) suggests that sensory region plays a pivotal role in proactive sense of ownership. In addition, the evidence that only the contralateral parietal is activated by the object approaching the participant's right hand further suggests that the predictive response is purely sensorial, rather than cognitive.

Our results enrich current models of body ownership suggesting that body ownership does not only arise reactively to multisensory information; rather, predictions are generated on what could be my body and what could not. This finding might have interesting implications in all those cases in which body representation is altered. One might think to alter multisensory body representations by altering the expectation that participants have about the morphological and structural features of their body. For instance, combining multisensory stimulation and expectation of a thin body in obese participants might produce a change in the way they experience their body. This is relevant as obese people have a distorted representation of the body (Scarpina et al., 2014), in that they tend to perceive themselves as thinner than they actually are. 


\section{Acknowledgements}

This study was supported by a grant from the BIAL Foundation to MC and FF (grant agreement 41/12; 'The body beyond the body').

\section{Conflict of interest}

The work described has not been published before. The authors declare no conflict of interest.

\section{Author contributions}

FF and MC conceived the study. All the authors implemented the study. EA and PP collected and analysed the data. FF and MC wrote the manuscript. All the authors commented on and approved the manuscript.

\section{Data accessibility}

All data created during this research are openly available at https:// osf.io/rxahg/?view_only=6bc23f0b70514a56a98ab5dc03cb5e31.

\section{References}

Ambrosini, E. \& Vallesi, A. (2016) Asymmetry in prefrontal resting-state EEG spectral power underlies individual differences in phasic and sustained cognitive control. NeuroImage, 124, 843-857.

Apps, M.A. \& Tsakiris, M. (2013) The free-energy self: a predictive coding account of self-recognition. Neurosci. Biobehav. R., 41, 85-97.

Arizono, N., Ohmura, Y., Yano, S. \& Kondo, T. (2016) Functional connectivity analysis of NIRS data under rubber hand illusion to find a biomarker of sense of ownership. Neural. Plast., 2016, 6726238.

Azanon, E., Longo, M.R., Soto-Faraco, S. \& Haggard, P. (2010) The posterior parietal cortex remaps touch into external space. Curr. Biol., 20, 1304-1309.

Ball, K. \& Sekuler, R. (1981) Cues reduce direction uncertainty and enhance motion detection. Percept Psychophys, 30, 119-128.

Blanke, O. (2012) Multisensory brain mechanisms of bodily self-consciousness. Nat. Rev. Neurosci., 13, 556-571.

Blanke, O. \& Mohr, C. (2005) Out-of-body experience, heautoscopy, and autoscopic hallucination of neurological origin Implications for neurocognitive mechanisms of corporeal awareness and self-consciousness. Brain Res. Brain Res. Rev., 50, 184-199.

Blanke, O., Landis, T., Spinelli, L. \& Seeck, M. (2004) Out-of-body experience and autoscopy of neurological origin. Brain, 127, 243-258.

Boas, D.A., Dale, A.M. \& Franceschini, M.A. (2004) Diffuse optical imaging of brain activation: approaches to optimizing image sensitivity, resolution, and accuracy. Neurolmage, 23(Suppl 1), S275-S288.

Botvinick, M. \& Cohen, J. (1998) Rubber hands 'feel' touch that eyes see. Nature, 391, 756.

Brigadoi, S., Ceccherini, L., Cutini, S., Scarpa, F., Scatturin, P., Selb, J., Gagnon, L., Boas, D.A. et al. (2014) Motion artifacts in functional nearinfrared spectroscopy: a comparison of motion correction techniques applied to real cognitive data. NeuroImage, 85(Pt 1), 181-191.

Brown, E.C. \& Brüne, M. (2012) The role of prediction in social neuroscience. Front. Hum. Neurosci., 6, 1-19.

Bubic, A., Von Cramon, D.Y. \& Schubotz, R.I. (2010) Prediction, cognition and the brain. Front. Hum. Neurosci., 4, 1-15.

Burbaud, P., Gross, C. \& Bioulac, B. (1985) Peripheral inputs and early unit activity in area 5 of the monkey during a trained forelimb movement. Brain Res., 337, 341-346.

Carlsson, K., Petrovic, P., Skare, S., Petersson, K.M. \& Ingvar, M. (2000) Tickling expectations: neural processing in anticipation of a sensory stimulus. J. Cogn. Neurosci., 12, 691-703.

Cooper, R.J., Selb, J., Gagnon, L., Phillip, D., Schytz, H.W., Iversen, H.K., Ashina, M. \& Boas, D.A. (2012) A systematic comparison of motion artifact correction techniques for functional near-infrared spectroscopy. Front. Neurosci., 6, 147.

Cope, M. \& Delpy, D.T. (1988) System for long-term measurement of cerebral blood and tissue oxygenation on newborn infants by near infra-red transillumination. Med. Biol. Eng. Comput., 26, 289-294.
Corbetta, M., Miezin, F.M., Dobmeyer, S., Shulman, G.L. \& Petersen, S.E. (1990) Attentional modulation of neural processing of shape, color, and velocity in humans. Science, 248, 1556-1559.

Costantini, M. (2014) Body perception, awareness, and illusions. Wiley Interdiscip. Rev.: Cogn. Sci., 5, 551-560.

Costantini, M. \& Haggard, P. (2007) The rubber hand illusion: sensitivity and reference frame for body ownership. Conscious. Cogn., 16, 229-240.

Cravo, A.M., Rohenkohl, G., Wyart, V. \& Nobre, A.C. (2013) Temporal expectation enhances contrast sensitivity by phase entrainment of low-frequency oscillations in visual cortex. J. Neurosci., 33, 4002-4010.

Cui, X., Bray, S. \& Reiss, A.L. (2010) Functional near infrared spectroscopy (NIRS) signal improvement based on negative correlation between oxygenated and deoxygenated hemoglobin dynamics. Neurolmage, 49, 30393046.

Cutini, S. \& Brigadoi, S. (2014) Unleashing the future potential of functional near-infrared spectroscopy in brain sciences. J. Neurosci. Meth., 232, 152156.

Duncan, A., Meek, J.H., Clemence, M., Elwell, C.E., Fallon, P., Tyszczuk, L., Cope, M. \& Delpy, D.T. (1996) Measurement of cranial optical path length as a function of age using phase resolved near infrared spectroscopy. Pediatr. Res., 39, 889-894.

van Ede, F., Jensen, O. \& Maris, E. (2010) Tactile expectation modulates pre-stimulus $\beta$-band oscillations in human sensorimotor cortex. NeuroImage, 51, 867-876.

van Ede, F., de Lange, F., Jensen, O. \& Maris, E. (2011) Orienting attention to an upcoming tactile event involves a spatially and temporally specific modulation of sensorimotor alpha- and beta-band oscillations. J. Neurosci., 31, 2016-2024.

Ehrsson, H.H. (2012). The Concept of Body Ownership and its Relation to Multisensory Integration. MIT Press, Cambridge, MA.

Ehrsson, H.H., Spence, C. \& Passingham, R.E. (2004) That's my hand! Activity in premotor cortex reflects feeling of ownership of a limb. Science, 305, 875-877.

Ehrsson, H.H., Holmes, N.P. \& Passingham, R.E. (2005) Touching a rubber hand: feeling of body ownership is associated with activity in multisensory brain areas. J. Neurosci., 25, 10564-10573.

Ferri, F. \& Costantini, M. (2016) Commentary: The magnetic touch illusion: a perceptual correlate of visuo-tactile integration in peripersonal space. Front. Hum. Neurosci., 10, 492.

Ferri, F., Chiarelli, A.M., Merla, A., Gallese, V. \& Costantini, M. (2013) The body beyond the body: expectation of a sensory event is enough to induce ownership over a fake hand. Proc. Biol. Sci. R. Soc., 280, 20131140.

Ferri, F., Costantini, M., Salone, A., Di Iorio, G., Martinotti, G., Chiarelli, A., Merla, A., Di Giannantonio, M. et al. (2014) Upcoming tactile events and body ownership in schizophrenia. Schizophr. Res., 152, 51-57.

Friston, K. (2010) Is the free-energy principle neurocentric? Nat. Rev. Neurosci., 11, 605-605.

Friston, K.J., Holmes, A.P., Worsley, K.J., Poline, J.P., Frith, C.D. \& Frackowiak, R.S.J. (1994) Statistical parametric maps in functional imaging: a general linear approach. Hum. Brain Mapp., 2, 189-210.

Friston, K., Kilner, J. \& Harrison, L. (2006) A free energy principle for the brain. J. Physiol.-Paris, 100, 70-87.

Gagnon, L., Cooper, R.J., Yucel, M.A., Perdue, K.L., Greve, D.N. \& Boas, D.A. (2012) Short separation channel location impacts the performance of short channel regression in NIRS. Neurolmage, 59, 2518-2528.

Guterstam, A., Zeberg, H., Ozciftci, V.M. \& Ehrsson, H.H. (2016) The magnetic touch illusion: a perceptual correlate of visuo-tactile integration in peripersonal space. Cognition, 155, 44-56.

Haggard, P., Taylor-Clarke, M. \& Kennett, S. (2003) Tactile perception, cortical representation and the bodily self. Curr. Biol., 13, R170-R173.

Halje, P., Seeck, M., Blanke, O. \& Ionta, S. (2015) Inferior frontal oscillations reveal visuo-motor matching for actions and speech: evidence from human intracranial recordings. Neuropsychologia, 79, 206-214.

Huppert, T.J., Diamond, S.G., Franceschini, M.A. \& Boas, D.A. (2009) HomER: a review of time-series analysis methods for near-infrared spectroscopy of the brain. Appl. Opt., 48, D280-D298.

Ionta, S., Gassert, R. \& Blanke, O. (2011a) Multi-sensory and sensorimotor foundation of bodily self-consciousness - an interdisciplinary approach. Front. Psychol., 2, 383.

Ionta, S., Heydrich, L., Lenggenhager, B., Mouthon, M., Fornari, E., Chapuis, D., Gassert, R. \& Blanke, O. (2011b) Multisensory mechanisms in temporo-parietal cortex support self-location and first-person perspective. Neuron, 70, 363-374.

Ionta, S., Martuzzi, R., Salomon, R. \& Blanke, O. (2014) The Brain Network reflecting Bodily Self-Consciousness: a functional connectivity study. Soc. Cogn. Affect. Neur., 9, 1904-1913. 
Kok, P., Failing, M.F. \& de Lange, F.P. (2014) Prior expectations evoke stimulus templates in the primary visual cortex. J. Cogn. Neurosci., 26, 1546-1554.

Longo, M.R., Schüür, F., Kammers, M.P.M., Tsakiris, M. \& Haggard, P. (2008) What is embodiment? A psychometric approach Cognition, 107, 978.

Lopez, C. (2015) Making sense of the body: the role of vestibular signals. Multisens. Res., 28, 525-557.

Lopez, C., Halje, P. \& Blanke, O. (2008) Body ownership and embodiment: vestibular and multisensory mechanisms. Neurophysiol. Clin., 38, 149161.

Lopez, C., Schreyer, H.M., Preuss, N. \& Mast, F.W. (2012) Vestibular stimulation modifies the body schema. Neuropsychologia, 50, 1830-1837.

Lunghi, C., Morrone, M.C. \& Alais, D. (2014) Auditory and tactile signals combine to influence vision during binocular rivalry. J. Neurosci., 34, 784-792.

MacKay, W.A. \& Crammond, D.J. (1987) Neuronal correlates in posterior parietal lobe of the expectation of events. Behav. Brain Res., 24, 167-179.

Makin, T.R., Holmes, N.P. \& Zohary, E. (2007) Is that near my hand? Multisensory representation of peripersonal space in human intraparietal sulcus. J. Neurosci., 27, 731-740.

Makin, T.R., Holmes, N.P. \& Ehrsson, H.H. (2008) On the other hand: dummy hands and peripersonal space. Behav. Brain Res., 191, 1-10.

Mesulam, M.M., Van Hoesen, G.W., Pandya, D.N. \& Geschwind, N. (1977) Limbic and sensory connections of the inferior parietal lobule (area PG) in the rhesus monkey: a study with a new method for horseradish peroxidase histochemistry. Brain Res., 136, 393-414.

Mihara, M., Miyai, I., Hattori, N., Hatakenaka, M., Yagura, H., Kawano, T., Okibayashi, M., Danjo, N. et al. (2012) Neurofeedback using real-time near-infrared spectroscopy enhances motor imagery related cortical activation. PLoS ONE, 7, e32234.

Okada, E., Firbank, M., Schweiger, M., Arridge, S.R., Cope, M. \& Delpy, D.T. (1997) Theoretical and experimental investigation of near-infrared light propagation in a model of the adult head. Appl. Optimizat., 36, 2131 .

Okamoto, M. \& Dan, I. (2005) Automated cortical projection of head-surface locations for transcranial functional brain mapping. Neurolmage, 26, 18 28

Olive, I., Tempelmann, C., Berthoz, A. \& Heinze, H.J. (2015) Increased functional connectivity between superior colliculus and brain regions implicated in bodily self-consciousness during the rubber hand illusion. Hum. Brain Mapp., 36, 717-730.

Pernet, C.R., Wilcox, R. \& Rousselet, G.A. (2012) Robust correlation analyses: false positive and power validation using a new open source matlab toolbox. Front. Psychol., 3, 606.

Perrett, D.I., Smith, P.A., Mistlin, A.J., Chitty, A.J., Head, A.S., Potter, D.D., Broennimann, R., Milner, A.D. et al. (1985) Visual analysis of body movements by neurones in the temporal cortex of the macaque monkey: a preliminary report. Behav. Brain Res., 16, 153-170.

Petersen, S.E., Robinson, D.L. \& Keys, W. (1985) Pulvinar nuclei of the behaving rhesus monkey: visual responses and their modulation. J. Neurophysiol., 54, 867-886.
Plichta, M.M., Herrmann, M.J., Baehne, C.G., Ehlis, A.C., Richter, M.M., Pauli, P. \& Fallgatter, A.J. (2007) Event-related functional near-infrared spectroscopy (fNIRS) based on craniocerebral correlations: reproducibility of activation? Hum. Brain Mapp., 28, 733-741.

Rizzolatti, G. \& Sinigaglia, C. (2016) The mirror mechanism: a basic principle of brain function. Nat. Rev. Neurosci., 17, 757-765.

Rousselet, G.A. \& Pernet, C.R. (2012) Improving standards in brain-behavior correlation analyses. Front. Hum. Neurosci., 6, 119.

Saenz, M., Buracas, G.T. \& Boynton, G.M. (2002) Global effects of featurebased attention in human visual cortex. Nat. Neurosci., 5, 631-632.

Scarpina, F., Castelnuovo, G. \& Molinari, E. (2014) Tactile mental body parts representation in obesity. Psychiat. Res., 220, 960-969.

Scholkmann, F., Spichtig, S., Muehlemann, T. \& Wolf, M. (2010) How to detect and reduce movement artifacts in near-infrared imaging using moving standard deviation and spline interpolation. Physiol. Meas., 31, 649-662.

Seltzer, B. \& Pandya, D.N. (1980) Converging visual and somatic sensory cortical input to the intraparietal sulcus of the rhesus monkey. Brain Res. 192, 339-351.

Serino, A., Alsmith, A., Costantini, M., Mandrigin, A., Tajadura-Jimenez, A. \& Lopez, C. (2013) Bodily ownership and self-location: components of bodily self-consciousness. Conscious. Cogn., 22, 1239-1252.

Shimada, S., Hiraki, K. \& Oda, I. (2005) The parietal role in the sense of self-ownership with temporal discrepancy between visual and proprioceptive feedbacks. Neurolmage, 24, 1225-1232.

Singh, A.K. \& Dan, I. (2006) Exploring the false discovery rate in multichannel NIRS. Neurolmage, 33, 542-549.

Singh, A.K., Okamoto, M., Dan, H., Jurcak, V. \& Dan, I. (2005) Spatial registration of multichannel multi-subject fNIRS data to MNI space without MRI. NeuroImage, 27, 842-851.

Stanton, G.B., Cruce, W.L., Goldberg, M.E. \& Robinson, D.L. (1977) Some ipsilateral projections to areas PF and PG of the inferior parietal lobule in monkeys. Neurosci. Lett., 6, 243-250.

Thakkar, K.N., Nichols, H.S., McIntosh, L.G. \& Park, S. (2011) Disturbances in body ownership in schizophrenia: evidence from the rubber hand illusion and case study of a spontaneous out-of-body experience. PLoS ONE, 6, e27089

Tsakiris, M. (2016) The multisensory basis of the self: from body to identity to others. Q. J. Exp. Psychol. (Hove), 70, 1-28.

Tsakiris, M. \& Haggard, P. (2005) The rubber hand illusion revisited: visuotactile integration and self-attribution. J. Exp. Psychol. Hum. Percept. Perform., 31, 80-91.

Tsakiris, M., Hesse, M.D., Boy, C., Haggard, P. \& Fink, G.R. (2007) Neural signatures of body ownership: a sensory network for bodily self-consciousness. Cereb. Cortex, 17, 2235-2244.

Wilcox, R. (2004) Inferences based on a skipped correlation coefficient. $J$. Appl. Stat., 31, 131-143.

Worsley, K.J. \& Friston, K.J. (1995) Analysis of fMRI time-series revisitedagain. Neurolmage, 2, 173-181.

Ye, J.C., Tak, S., Jang, K.E., Jung, J. \& Jang, J. (2009) NIRS-SPM: statistical parametric mapping for near-infrared spectroscopy. Neurolmage, 44, $428-447$. 